\title{
Emerging and Analysis Trends of SOA and SAAS
}

\author{
Sonia Kumari \\ Faculty of Information Technology \\ Harlal Institute of management and Technology, \\ Greater Noida
}

\begin{abstract}
There are some issues of mystification between the concepts of Service Oriented Architecture (SOA) and Software as a Service (SaaS). This paper aims to discover the concepts of SOA and SaaS in order to give better perceptive of technologies and this paper search different type of approaches and techniques that address the testing of SOA and Saas based services.
\end{abstract}

\section{Keywords-SOA, Saas, SOA Testing, Testing Perspectives}

\section{INTRODUCTION}

The service-oriented architecture (SOA) supports service orientation. Service-Orientation represents a way of thinking about business and IT. The service-oriented architecture (SOA) removed the gap between software and business. Now a days, there is a business transformation among enterprises and they adopt a service based IT model. SOA provides solutions for integrating various systems that support interoperability, movable coupling and reuse. To accomplish clients need one service appeal to another services. It is possible that there is some development among these external services[1]. This dynamic, vibrant and adaptive nature of SOA makes major concerns about its reliability and defect free implementation. So, testing is necessary for SOA based application. However, the SOA testing approach is completely different from Saas testing approach. SaaS testing also acquired a remarkable place by providing various services on clouds. There is offline regression testing and stationary test case profiling. But, SOA testing approach supports collaborative testing. Here, verification is done among the service suppliers, service brokers and clients in a combined way. In SOA testing, testing location should be remote, circulated, multi-phase and multi-agent. SOA testing supports online regression testing where information collected dynamically. Reliability is ensured by dynamic profiling and cluster testing. It composed of loosely coupled, discoverable, reusable and interoperable services. SOA is an approach for designing controlling and deploying systems that represent reusable business functionality. Web services are used to implement the SOA in which service interface describes using the web service description language (WSDL) and extensible markup language (XML). The message is transmitted using simple object access protocols (SOAP) over hypertext transfer protocol (HTTP). The web service is based on open standards such as HTTP and XMLbased protocols including SOAP and WSDL, Web services are hardware, programming language, and operating system independent . Web services are formally and fully described by an XML-based WSDL document. The best method to approach a WSDL document is to understand that different XML elements take responsibility for describing different levels of detail. For instance, the element is a detailed listing of the types that factor into a given message. On the other hand, the element simply lists the messages that factor into a given operation without going into any detail as to what these communication seem like.

\section{SOFTWARE AS A SERVICE(SAAS)}

Software as a service (SaaS) is a software supply model in which a moderator Supplier hosts applications and makes them available to customers over the Internet. SaaS is one of three categories of cloud computing, along with infrastructure as a service (IaaS) that provides virtualized computing resources over the internet and these can also include server, storage, network, security and system management and platform as a service (PaaS) is another category of cloud computing model in which a moderator provider delivers hardware and software tools usually those needed for application development to users over the internet and these can also include web, Application, database[9]. Moreover, SaaS is a centralized, configurable, scalable and multitenant system which uses meta-data to provide support for different customers with different requirements at the same time. SaaS is changing the way in which people construct, sell, purchase and utilize the software that's why it has significant impact on software industry. On the technical area, the SaaS supplier deploying patches and upgrades to the application visibly and delivering contact to end users over the Internet through a browser or smart-client application. Suppliers might even provide tools that allow users to change the workflow, information representation and supplementary aspects of the application's operation for their use.

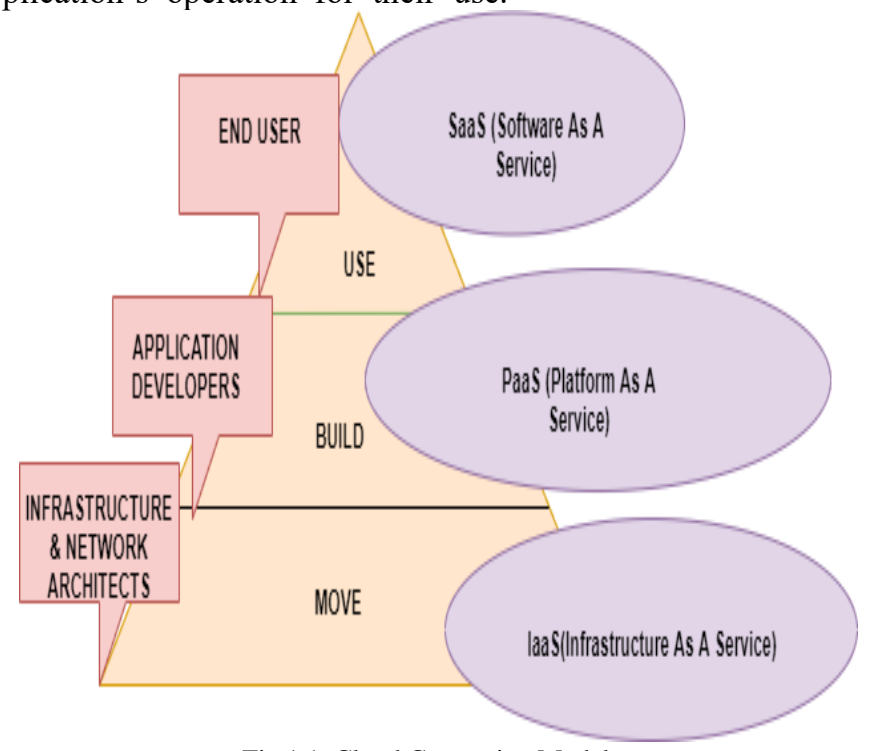

Fig 1.1. Cloud Computing Model 


\section{SERVICE-ORIENTED ARCHITECTRE (SOA)}

Service-Oriented Architecture (SOA) is an architectural approach in which applications make use of services accessible in the network. In this architecture approach, services are provided to figure applications, through a communication call over the internet. In reality, Service Oriented Architecture (SOA) presents solutions as services in an architectural model. Services in SOA form a collection of independent functions which are more related to the service interface rather than relating to each other. In SOA architecture, the client switches to another tasks while is waiting for the reply but in traditional client- server architectures the client should wait idly for its turn to be served.

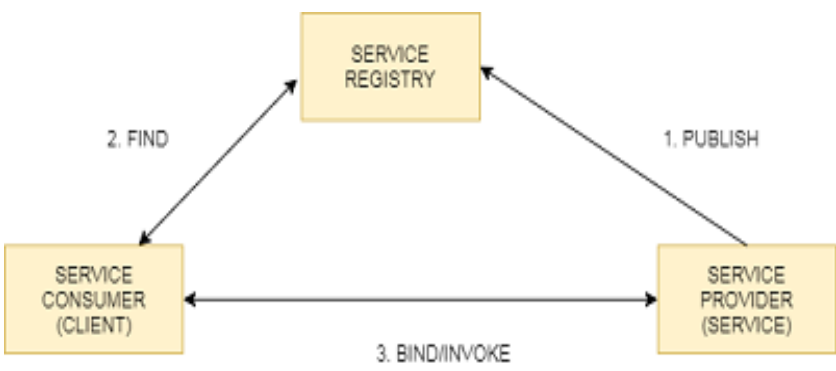

Fig 1.2 Service-Oriented Architecture

\section{SOA TESTING}

This part describe diverse SOA Testing concept like SOA testing perspectives, SOA testing levels. These concepts are described as follow

\section{A. SOA Testing Perspectives}

SOA imposes different need and challenges to different shareholder involves in the testing activities. Shareholder interested to make sure the originality of service behavior during its lifetime. Different testing perspectives are detailed below: .

- Supplier/Developer perspective: Services are built by service developer and he deliver both the interface and implementation of services. In order to announce a highly reliable service, service developer need to test the services. Developer derived the test cases but it may not imitate real tradition scenarios. Service Supplier tests the services that meet the service level agreement (SLA) agreed upon with the customer. Black box testing technique is used by the service Supplier .

- Provision Integrator's Perspective: Now service testing is done during the design phase in order to discover the functional and non-functional assumptions. Existing services are used by the service Integrator to create an end-user application. The focus of Integrator perspective is on business need rather than implementation facts which command several services incantation.

- Moderator/ Certifier Perspective: Service Integrator employed third parties for the assessment of faultproneness of a service. The trusted moderator helps to reduce the number of shareholders and resources involved in the testing activities. The thirdparty/ certifier testing perspective is different from the Service Integrator's perspective .

- End-user Perspective: Service-oriented system is used by end-users. End-users do not be familiar with the technology of service testing. The end-users do not have any scope for service testing, the only concern is that the system they apply work accurately.

\section{SOA TESTING LEVELS}

We can describe different types of testing in SOA based application are as follows:-

a)Unit Testing: Unit testing is also known as servicecomponent-level testing. Developer performed the unit testing to guarantee that component functionality within the services work accurately. In unit testing a smallest piece of software is tested. This smaller piece is isolated from the remainder of the code. Now, the developer checks the behavior of this piece and to ensure it behaves exactly as we expected or not. By service integration each module must be tested .

b) Integration Testing: In this testing phase our focus is on service interfaces. Integration testing ensures the interface behavior and sharing information between the services as specified. This test phase may include testing peripheral services to organizations .

c) System Testing: System testing test the SOA technical result and to guarantee business condition and definite business acceptance criteria that have to be met. In this testing phase there is a requirement to recognize the quality and test coverage of the previous test phases and this ensure the testing of key business scenario.

d)Regression Testing: Regression testing of an SOA system guarantees that no previously working services fail as a solution of modification or conservation. This testing phase determines the service up to point of repair has not been affected by the fixes[3]. So, Regression testing is also wellknown as validation testing.

\section{DIFFERENCE BETWEEN SOA AND SAAS}

SaaS services is provided to the customers through web services only . SOA provides a tool to organize and quickly re-configure as business circumstances change the applications and databases owned by a company [5].

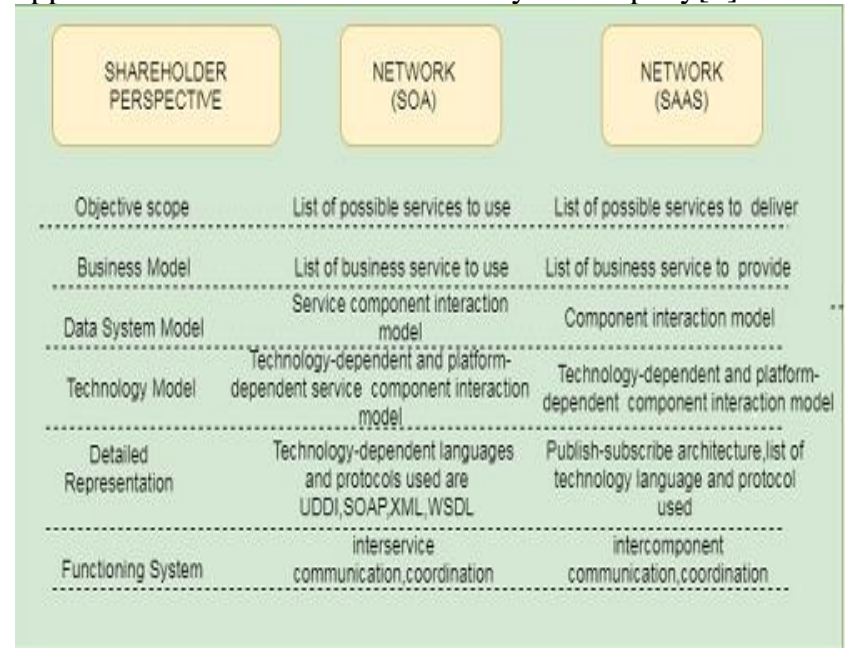

Fig 1.3 SOA Versus SAAS 


\section{CONCLUSION}

This paper offered various testing perspectives and various testing level of SOA based services. Further, we also discussed SOA and SaaS differences. Another aspect of this paper is to identify diverse testing problems presented in different testing approaches. The people who work with SOA concepts when they come to the SaaS cloud, they misinterpret the concepts. Compare to SOA architecture based on reusability for business while SaaS is based on the reusability for service[8]. SaaS is supported by SOA.SaaS is software delivery model whereas SOA is software construction model. This study has been assessed the concepts of SaaS and SOA. In future researches may concentrate more on the characteristics of the services used in SaaS and SOA, their quality of service and also other new technologies based on the services.

\section{ACKNOWLEDGMENT}

I express our greatest gratitude and appreciation of Department of Computer Science, University of Delhi and Harlal institute of management and technology, Greater Noida.

\section{REFERENCES}

[1] Askarunisa, A., A. M. Abirami, K. Arockia Jackulin Punitha, B. Karthik Selvakumar, and R. Arun kumar. "Sequence-based techniques for black-box testing and test case prioritization for composite service testing".

[2] Youssef Bassil. Distributed, cross-platform and regression testing architecture for service-oriented architecture(SOA).

[3] Rajanikanta Mohanty, Binod Kumar Pattanayak, Bhagabat Puthal and Durgaprasad Mohapatra. A Road Map to Regression Testing

[4] Service-Oriented Architecture(SOA) Based Applications. In Journal of theoretical and applied information technology (2012), vol. 36.

[5] https://medium.com/@invxtech/the-difference-between-saas-softwareas-a-service-and-soa-service-oriented-architecture-431cb218a09

[6] Rahmansyah, R. and F. Gaol, 2013. Service oriented architecture governance implementation in a software development project as an enterprisesolutions.J.Comput.Sci.,9:1638-1647. DOI:10.3844/jcssp.2013.1638.1647.

[7] SaaS, 2009. SaaS: The future of flexible software model.

[8] Tang, G., 2011. The SAAS architectures and design on the five layers driving model. Manage. Eng., 2: 61-65.

[9] https://searchcloudcomputing.techtarget.com/definition/Infrastructureas-a-Service-IaaS 